\title{
A Comprehensive Bioinformatics Analysis of the Nudix Superfamily in Arabidopsis thaliana
}

\author{
D. Gunawardana, ${ }^{1,2}$ V. A. Likic, ${ }^{1}$ and K. R. Gayler ${ }^{1}$ \\ ${ }^{1}$ Department of Biochemistry and Molecular Biology, Bio21 Molecular Science and Biotechnology Institute, \\ University of Melbourne, Parkville, Victoria 3010, Australia \\ ${ }^{2}$ School of Biological Sciences, University of Sydney, New South Wales 2006, Australia
}

Correspondence should be addressed to D. Gunawardana, dilanthag_12@yahoo.com.au

Received 15 October 2008; Accepted 16 April 2009

Recommended by H. Heng

Nudix enzymes are a superfamily with a conserved common reaction mechanism that provides the capacity for the hydrolysis of a broad spectrum of metabolites. We used hidden Markov models based on Nudix sequences from the PFAM and PROSITE databases to identify Nudix hydrolases encoded by the Arabidopsis genome. 25 Nudix hydrolases were identified and classified into 11 individual families by pairwise sequence alignments. Intron phases were strikingly conserved in each family. Phylogenetic analysis showed that all multimember families formed monophyletic clusters. Conserved familial sequence motifs were identified with the MEME motif analysis algorithm. One motif (motif 4) was found in three diverse families. All proteins containing motif 4 demonstrated a degree of preference for substrates containing an ADP moiety. We conclude that HMM model-based genome scanning and MEME motif analysis, respectively, can significantly improve the identification and assignment of function of new members of this mechanistically-diverse protein superfamily.

Copyright ( 2009 D. Gunawardana et al. This is an open access article distributed under the Creative Commons Attribution License, which permits unrestricted use, distribution, and reproduction in any medium, provided the original work is properly cited.

\section{Introduction}

Nudix hydrolases are a diverse superfamily of pyrophosphatases found in all three domains_-archaea, bacteria, and eukarya $[1,2]$. More than 1800 open reading frames coding for Nudix hydrolases, in over 360 different species, have been identified by bioinformatics searches [3]. Members of the superfamily of Nudix hydrolases predominantly catalyze the hydrolysis of a wide range of small nucleotide substrates composed of a $n u$ cleoside di phosphate linked to another moiety $X$, hence the name Nudix [4]. Nucleoside triphosphates (Npp-p), dinucleoside polyphosphates (Npp$\mathrm{p}(\mathrm{n}) \mathrm{N})$, nucleotide sugars (Npp-sugar), and mRNA (NpppRNA) are examples of such Nudix compounds.

The Nudix hydrolases constitute a "mechanistically diverse superfamily". This by definition is a group of evolutionarily related proteins that utilize a common mechanistic attribute, such as a specific partial reaction, intermediate, or transition state to catalyze different overall reactions with diverse substrate specificities [5]. In line with this definition, all members of the Nudix superfamily share a conserved amino acid sequence termed the Nudix box, the signature motif for Nudix hydrolases [1, 4]. It constitutes a key component of the catalytic site of these enzymes [4]. The Nudix motif, as defined by Bessman et al. [4], is comprised of 23 amino acids represented by the sequence $\mathrm{G}(\mathrm{X} 5) \mathrm{E}(\mathrm{X} 7) \mathrm{REUXEEXGU}$, where $\mathrm{U}$ denotes a bulky hydrophobic amino acid, and $\mathrm{X}$ depicts any amino acid. Structural studies combined with site-directed mutagenesis have demonstrated that the Nudix box contains conserved residues essential for substrate hydrolysis [6-8]. Hydrolysis is mediated through nucleophilic substitution at $\beta$-phosphorus and is independent of the type of substrate cleaved [6-9]. Two glutamates, three residues apart, within an essential and conserved REXXEE sequence motif within the Nudix box, play mandatory roles as the effector of catalysis and as a ligand to the enzyme bound metal ion cofactor, respectively [6-9].

In vivo studies have demonstrated the importance of individual Nudix enzymes in plants. Overexpression of gene At4g11980, encoding an ADP-ribose/ADP-glucose pyrophosphatase, reduced levels of both its substrate ADPglucose and the biopolymer starch in Arabidopsis plants [10]. 
Inactivation of a fibroblast growth factor type Nudix enzyme (FGFTNE) gene (At4g12720) in Arabidopsis plants led to a pleiotropic phenotype with dwarfed growth, curled leaves, microscopic cell death, higher resistance to pathogens, and increased levels of reactive oxygen species (ROS) [11]. A T-DNA knockout of the same FGFTNE gene resulted in growth retardation, increased basal resistance to virulent Hyaloperonospora parasitica, and fourfold higher levels of the defense molecule salicylic acid, suggesting a deregulated defense mechanism [12]. T-DNA knockout of the Nudix gene At5g13570 coding for the Arabidopsis mRNA decapping enzyme similarly demonstrated its importance for plant growth and elongation [13]. Heterozygous mutation of At5g13570 resulted in stunted hypocotyls and roots, and the homozygous knockout was postembryonically lethal [13].

In spite of phenotypical evidence from gene inactivation studies and the characterization in vitro of selected plant Nudix hydrolases, understanding of the diversity and evolution of this enzyme superfamily in plants is incomplete. The most comprehensive bioinformatics analysis of the Nudix superfamily performed so far was restricted to three families of Nudix hydrolases and was based on sequence alignments alone [14]. With the exception of the human and yeast Nudix hydrolases [15], knowledge of the diversity of Nudix hydrolases in eukaryotic organisms, particularly plants, is limited. The first plant genome to be sequenced was that of A. thaliana, and genome resources on this organism are freely available for bioinformatics analyses [16]. We have undertaken an extensive analysis of the Nudix superfamily in A. thaliana to (1) assess the diversity of Nudix hydrolases in plants by gene identification and annotation, (2) classify families of enzymes within the Nudix superfamily based on sequence conservation, genetic architecture, and phylogenetic proximity, (3) identify motifs that could determine substrate specificities and functional classes of enzymes, and (4) analyze motifs to predict substrate specificities associated with particular motifs. It is hoped that a bioinformatics analysis incorporating sequence information, gene structures, evolutionary relationships, and motifs of putative functional significance will aid future genome/proteome studies of plant Nudix hydrolases, to identify Nudix genes and their encoded proteins from sequenced genomes and to facilitate the engineering of functional motifs for protein structurefunction studies.

\section{Materials and Methods}

2.1. Hidden Markov Model Scans. The Arabidopsis proteome was scanned for putative Nudix hydrolases in three analytical steps. Initially the conceptual proteome of A. thaliana was scanned with hidden Markov models (HMMs) [17] based on the "seed" and "full" models for the superfamily of Nudix hydrolases from the PFAM database. The total non-redundant sequences from both the "seed" and "full" model based searches were combined. A second analysis of the Arabidopsis conceptual proteome was performed using HMM models derived from the two-element fingerprint (two consensus sequence motifs of 15 and 16 residues) that provides a signature for the Nudix box in the PROSITE database. In all HMM scans the cutoff $E$-value was 0.01 . The total entries that matched the search criteria from the two searches (using the PFAM and PROSITE sequences) were pooled. The non-redundant pooled sequences were further reduced to those containing the hexaresidue motif REXXEE, a region conserved and essential within the active site of Nudix hydrolases. Finally, sequences not containing the 23 residue Nudix box consensus G(X5)E(X7)REUXEEXGU were discarded. This final selection was by individual manual scrutiny.

2.2. Multiple Sequence Alignment of Protein and DNA Sequences. Multiple protein sequences were aligned with the European Bioinformatics Institute (EBI) CLUSTALW server and the CLUSTALW algorithm [18] implemented within Data Analysis in Molecular Biology and Evolution 4.13 (DAMBE) [19]. For the alignment of DNA sequences, the corresponding protein sequences were first aligned using the CLUSTALW algorithm and subsequently converted to the respective DNA sequences.

2.3. Determination of Genetic Architecture and Intron Phases. Intron-exon architecture and intron phases for each Nudix hydrolase gene were obtained from the Arabidopsis Information Resource (TAIR) [20] and the Xpro database [21].

2.4. Phylogenetic Analysis. Maximum parsimony trees for the Arabidopsis Nudix hydrolases were created using DAMBE version 4.13 [19]. The relationships between adjacent nodes were based on bootstrap support from 500 replicates. The gene trees were created either as unrooted trees or considering the gene At1g68760 as the outgroup. The gene product of Atlg68760 catalyzes an essential step in the folate synthesis pathway, only fully conserved in plants and bacteria [22].

2.5. Motif Analysis. The program MEME version 3.0.4 was used for the elucidation of motifs in Nudix sequences [23]. MEME was run locally with the parameters set to their default values. The model "zoops" was used, and the motif widths were constrained to between 6 and 90 residues.

2.6. Homology Modeling. Homology modeling of the threedimensional structures of proteins was performed using SWISS-MODEL [24], and the structures further analyzed using PyMOL version 0.97.

2.7. Assessment of Gene Duplication Events. The Nudix sequences from five genomes (Synechococcuselongatus, Saccharomycescerevisiae, Homosapiens, Oryzasativa, and A. thaliana) comprising of a broad taxonomical spectrum were used to create a gene tree. The genomes were representative of cyanobacteria, unicellular eukaryotes, mammals, and monocotyledonous and dicotyledonous plants. The protein sequences of the Nudix hydrolases from the above organisms were first downloaded from the PFAM database, then aligned using the CLUSTAL W algorithm [18], and finally reverse translated into the corresponding DNA sequences. The gene 
tree was created by maximum parsimony analysis using DAMBE version 4.13 [19]. Adjacent nodes were derived from bootstrap support from 250 replicates. The binary species tree for the corresponding organisms was downloaded from the NCBI taxonomy server. The gene trees were reconciled to the species tree using NOTUNG 2.0 beta using the default parameters [25].

\section{Results}

3.1. Identification of Nudix Hydrolases in A. thaliana. The identification of all members of the superfamily of Nudix hydrolases in A. thaliana was sought using a hidden Markov model-(HMM-) based approach to detect the widest possible array of related sequences including many potential false positives (Figure 1(a)). The scanning of the Arabidopsis proteome for these putative Nudix hydrolases was first conducted using the sequence resources of the PFAM database [26]. The HMMs based on "seed" and "full" alignments for the superfamily of Nudix hydrolases from the PFAM database were used to scan the Arabidopsis proteome with the $E$-value cutoff of 0.01 . The $E$-value cutoff of 0.01 is arbitrary, but a typical one used in BLAST and HMM searches. There were 61 hits for the "seed", and 74 hits for the "full" model. The intersection of two sets of sequences (a non-redundant set) contained 93 sequences and was taken as the first set of putative Nudix hydrolases (Figure 1(a)).

In a second step, HMMs for the Nudix superfamily were built with the aid of the signature motifs characteristic of Nudix hydrolases within the PROSITE database [27, 28]. In PROSITE, the Nudix hydrolases are represented by a two-element fingerprint (two consensus motifs of 15 and 16 residues) that provides a signature for the Nudix hydrolase superfamily (PROSITE accession code PS00502, Nudix hydrolase family motifs II-4 and I-4). The two motifs span the full length of the Nudix (MutT) domain and include the region encoded by the PROSITE pattern MUTT (PS00893). The motifs (I and II) which form the two-element fingerprint can be described by the consensus sequences "(X5)G(X5)E(X3)" and "(X5)REUXEEXGU(X2)", respectively, where $U$ denotes a hydrophobic residue, and $\mathrm{X}$ specifies any residue, with an overlap between the final residue of motif I and first residue of motif II. The HMMs built from the two PROSITE motifs were used to scan the A. thaliana conceptual proteome to identify putative Nudix enzymes. There were a total of 151 unique hits matching the two HMMs with the $E$-value <.01 (Figure 1(a)).

The entries that matched the search criteria using the PFAM-based search (93 sequences) and PROSITE-based search (151 sequences) were pooled, and the non-redundant sequences (intersection of the two sets, a total of 103 sequences) retained for further analysis. These sequences were further scrutinized for the presence of the REXXEE motif, a region conserved within the active site of Nudix hydrolases [15]. This hexaresidue consensus contains two essential glutamates (REXXEE) that are central components of the catalytic mechanism-the first acts as the effector of catalysis that deprotonates the attacking water molecule, whereas the second provides a ligand to the enzyme-bound
(1) The HMMs of the seed and full alignments for the superfamily of Nudix hydrolases from the PFAM database were used to scan the proteome of $A$. thaliana $(E$-value $<.01)$; the total non-redundant sequences from both the seed and full alignment based searches were considered for identification purposes 93 sequences

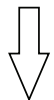

(2) HMM model for Nudix hydrolases derived from the two-element fingerprint for Nudix hydrolase in the PROSITE database was used to scan the proteome of $A$. thaliana $(E$-value $<.01)$ 151 sequences

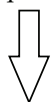

(3) The combined non-redundant matches from the dual searches were further scrutinized with the conserved sequence 'REXXEE' to narrow the search to exact Nudix hydrolases 26 sequences

Final manual analysis established the presence of 25 Nudix hydrolases encoded by the Arabidopsis genome

(a)

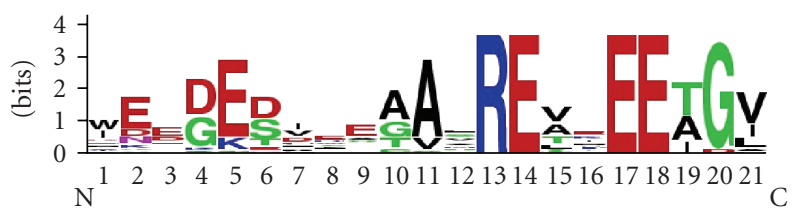

(b)

Figure 1: Identification of Nudix hydrolases encoded by the Arabidopsis genome. (a) Flowchart by which putative Nudix proteins encoded by the Arabidopsis genome were identified within the PFAM and PROSITE databases. (b) Consensus for the final 21 residues of the Nudix motif within the identified members of the Nudix superfamily in A. thaliana.

metal ion cofactor [9]. This eliminated all but 26 sequences (Figure 1(a)).

Further manual analysis for the presence of the complete 23 residue Nudix box signature sequences (G(X5)E(X7)REUXEEXGU) reduced the total number to 25 Nudix hydrolases. Figure 1(b) shows the extent of conservation of these residues amongst these 25 Nudix hydrolases. As demonstrated in Figure 1(b), in spite of the essential conservation of the REXXEE sequence, subtle variations to the 23 residue consensus were present in identified members of the Nudix superfamily, mainly in residues not directly involved in the mechanism of catalysis.

For this study, the Nudix superfamily was defined by and limited to genes encoding a complete 23 residue signature sequence. Nevertheless the search for Nudix hydrolases within the Arabidopsis genome was extended to check for GDP-mannose mannosyl hydrolases, a Nudix family where the REXXEE signature sequence is absent. Direct BLASTP searches were carried out using the E. coli GDP-mannose mannosyl hydrolase against the Arabidopsis proteome. However, not a single putative GDP-mannose mannosyl hydrolase was detected. The absence of GDP-mannose mannosyl 
TABLe 1

\begin{tabular}{lc}
\hline Pattern & A. thaliana matches \\
\hline G-x(5)-E-x(7)-R-E-x(2)-E-E-x-G-x & 107 \\
G-x(5)-E-x(7)-R-E-[VILMFWC]-x-E-E- & 62 \\
$x-G-[V I L M F W C]$ & \\
\hline
\end{tabular}

hydrolases in A. thaliana was consistent with the restriction of this family entirely to gram-negative bacteria.

As a separate measure of verification, the 23 residue Nudix box sequence patterns were utilized to search the NCBI A. thaliana database. The patterns shown in Table 1 were used to search the database using the Seedtop program from NCBI.

The total complement of Nudix hydrolase sequences (25) identified using HMMs were not found within the 62 and 107 sequences identified by pattern matching. The products of genes At1g28960, At2g04430, At4g11980, and At5g45940 were not present in both sequence listings (data not shown) demonstrating the limitation of this method to identify the total compliment of Nudix hydrolases encoded by the Arabidopsis genome. Therefore, we conclude that an organized HMM-based analysis, as employed in this study, is a better alternative to pattern matching for the identification of members of this enzyme superfamily.

3.2. Classification and Annotation of Families of Enzymes within the Nudix Superfamily. Nudix hydrolases constitute a superfamily, as defined in the Structural Classification of Proteins Database (SCOP) where proteins with low sequence identity but whose structures and functional features suggest a common evolutionary origin are classified as a superfamily [29]. In this context, Nudix hydrolases possess a common fold $(\alpha+\beta$ fold $)$ and a conserved mechanism of catalysis (substitution at $\beta$-phosphorus) but relatively low overall sequence similarity to each other. By contrast, the term "family" as defined by SCOP is restricted to a group of proteins that have both a common evolutionary origin and residue identities of $30 \%$ or greater [29]. Therefore, the scores of pairwise alignments were used as the foundation for the classification of families within the Nudix superfamily with a threshold score of 30 assigned as the cutoff value. Pairwise alignment scores were calculated as the number of identities in the best alignment divided by the number of residues compared, excluding gap positions and presented as percent identity scores.

Table 2 lists the families where sequence identity was $>30 \%$. The full matrix of pairwise sequence alignment scores is presented as in Supplementary Table A in Supplementary Material available online at doi: 10.1155/2009/820381. The nomenclature used in this paper is derived from gene identities as annotated in the TAIR database and not their protein counterparts, since for five of the Nudix hydrolases (At1g30110, At3g10620, At5g06340, At5g13570, At4g25440) no identity has been presented using the convention for nomenclature of Arabidpopsis Nudix proteins (PrefixAtNUDT). For consistency purposes, the Nudix genes/gene products from $A$. thaliana will be identified in the standard
TABle 2: The putative Nudix hydrolases identified by the HMM based approach. The locus ID, the designated protein and their assigned families are shown.

\begin{tabular}{|c|c|c|}
\hline Gene ID & $\begin{array}{c}\text { Nomenclature of protein } \\
(\mathrm{NCBI})\end{array}$ & Family \\
\hline Atlg30110* & - & $\mathrm{Ap}_{n} \mathrm{~A}$ hydrolase \\
\hline At3g10620* & - & $\mathrm{Ap}_{n} \mathrm{~A}$ hydrolase \\
\hline At5g06340* & - & $\mathrm{Ap}_{n} \mathrm{~A}$ hydrolase \\
\hline Atlg12880* & AtNUDT12 & DIPP \\
\hline Atlg14860 & AtNUDT18 & DIPP \\
\hline Atlg73540* & AtNUDT21 & DIPP \\
\hline Atlg18300* & AtNUDT4 & DIPP \\
\hline At2g01670 & AtNUDT17 & DIPP \\
\hline At3g12600* & AtNUDT16 & DIPP \\
\hline At3g26690 & AtNUDT13 & DIPP \\
\hline At1g28960 & AtNUDT15 & $\begin{array}{l}\text { Coenzyme A } \\
\text { pyrophosphatase }\end{array}$ \\
\hline At2g33980 & AtNUDT22 & $\begin{array}{l}\text { Coenzyme A } \\
\text { pyrophosphatase }\end{array}$ \\
\hline At5g45940 & AtNUDT11 & $\begin{array}{l}\text { Coenzyme A } \\
\text { pyrophosphatase }\end{array}$ \\
\hline At2g04430 & AtNUDT5 & FGFTNE \\
\hline At2g04450* & AtNUDT6 & FGFTNE \\
\hline At4g12720* & AtNUDT7 & FGFTNE \\
\hline At5g $47240^{*}$ & AtNUDT8 & FGFTNE \\
\hline At5g47650 & AtNUDT2 & FGFTNE \\
\hline Atlg68760* & AtNUDT1 & $\begin{array}{l}\text { Dihydroneopterin } \\
\text { triphosphate hydrolase }\end{array}$ \\
\hline Atlg79690* & AtNUDT3 & $\begin{array}{l}\text { Isopentenyl diphosphate } \\
\text { isomerase }\end{array}$ \\
\hline At2g42070 & AtNUDT24 & Unknown \\
\hline At4g11980 & AtNUDT14 & $\begin{array}{l}\text { ADP-ribose } \\
\text { pyrophosphatase }\end{array}$ \\
\hline At5g13570* & - & mRNA decapping enzyme \\
\hline At5g20070* & AtNUDT19 & NADH hydrolase \\
\hline At4g25440 & - & Unknown \\
\hline
\end{tabular}

* Nudix genes amplified by PCR from the Arabidopsis cDNA library.

format for chromosome-based nomenclature for Arabidopsis genes as described in the TAIR database throughout this publication.

Six proteins failed to align at a level $>30 \%$ to any other protein identified from HMM-based proteome scanning (Supplementary Table A) and were designated as those represented by a single family member. The functional classification of each family, as listed in Table 2, was based on similarities to enzymes of known substrate preferences from other species. BLASTP searches were carried out in this 

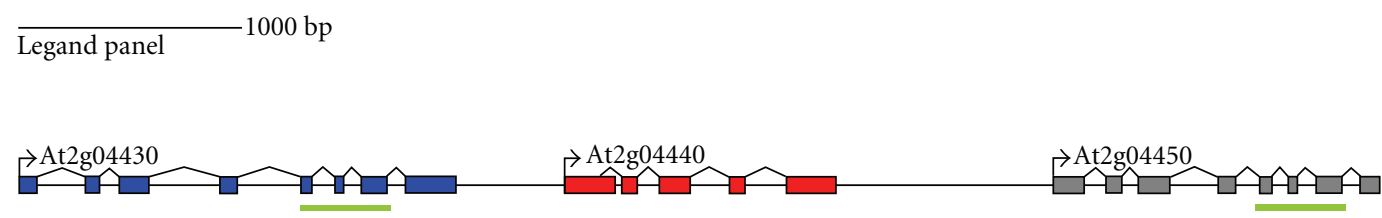

(a)

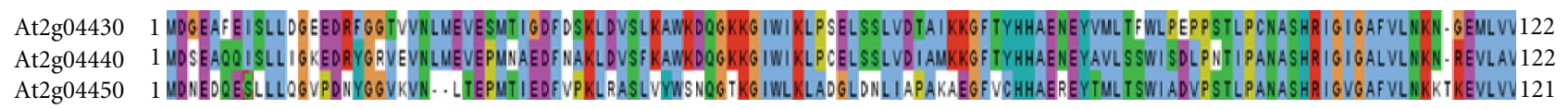

Nudix box

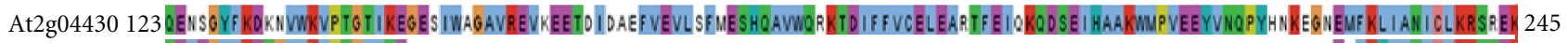

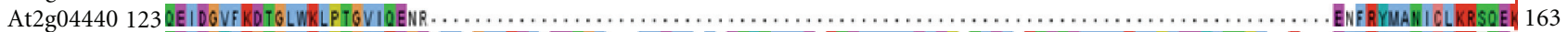

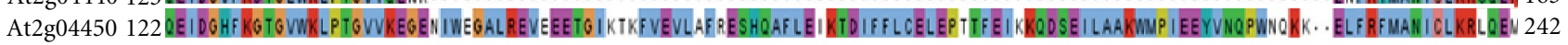

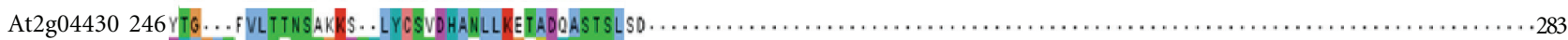

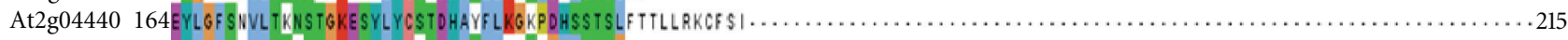

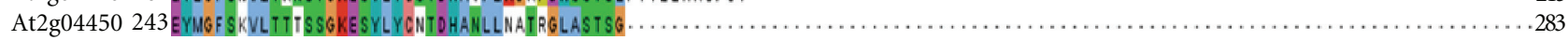

(b)

Figure 2: Architecture of a putative FGFTNE pseudogene. (a) Intron-exon architecture of the At2g04430, At2g04440, and At2g04450 genes. (b) The sequence alignment of the gene products of At2g04430, At2g04440, and At2g04450. The exons that are missing in the At2g04440 gene and the analogous protein sequence are underlined in green. The Nudix box region is also marked.

study on each of the gene products listed in Table 2 , and a $>30 \%$ sequence identity level to enzymes of known function was used as the basis for their annotation. The annotations assigned in this way agreed with those in the NCBI database.

In all, nine protein families were assigned functions, and there were two further families of unknown function. The nine designated families were $\mathrm{Ap}_{n} \mathrm{~A}$ hydrolases, diphosphoinositol polyphosphate phosphohydrolases (DIPPs), FGFTNEs, coenzyme A pyrophosphatases, NADH hydrolases, dihydroneopterin triphosphate phosphohydrolases (DHNTPs), ADP-ribose pyrophosphatases, isopentenyl diphosphate isomerases, and mRNA decapping enzymes (Table 2). The designation of FGFTNEs was based on similarity of these proteins to a protein fragment encoded by the human fibroblast growth factor mRNA. In this study, this family has been designated as FGFTNEs, due to both the similarity of these proteins to type 2 fibroblast growth factors and the clear presence of the signature Nudix motif in all familial members.

One pseudogene, with high sequence identity to the FGFTNEs, was also identified in the preliminary HMMbased scanning of the Arabidopsis proteome. This pseudogene which is annotated as At2g04440 is found between two putative Nudix genes At2g04430 and At2g04450, which are located within a short stretch of $6 \mathrm{~kb}$ of the genome (Figure 2). Genes At2g04430 and At2g04450 are both putative FGFTNEs with $58 \%$ sequence identity to each other at the protein level. The encoded protein of the pseudogene At2g0440 also shows significant overall similarity to all other FGFTNEs. However the At2g04440 gene product failed to appear amongst the 25 Nudix hydrolases determined by the HMM-based scanning of the Arabidopsis proteome, due to the absence of a region of 84 residues inclusive of the Nudix box and hence the catalytic site (Figure 2). It is likely that the Arabidopsis genome contains other pseudogenes which were not identified in this study due to their more extensive sequence divergences. By contrast, it is unlikely that any of 25 Nudix genes identified in this study are pseudogenes. Evidence for expression of the genes as mRNAs was sought by PCR-based screening of an Arabidopsis cDNA library created from mRNA of 42 day old $A$. thaliana Col- 0 ecotype plants. 14 of the 25 Nudix genes identified in this study were tested. All 14 were detected as cDNAs in the Arabidopsis cDNA library (Table 2) confirming that all 14 genes were capable of expression at the mRNA level. The expression level data were limited to the above genes since the cDNAs of these 14 genes were used for their initial cloning in to maintenance/expression vectors and for the production of selective proteins in bacterial expression systems, for subsequent enzymological studies. The characterization of one of the proteins, the mRNA decapping enzyme (gene product At5g13570), has already been published in the journal NucleicAcidsResearch [30].

3.3. Analysis of Intron-Exon Architecture. Genes of each of the families of the Nudix superfamily showed a high degree of conservation of intron-exon architecture. Introns have been mapped and characterized in most genomes of model organisms including $A$. thaliana. Spliceosomal introns have been classified based on the position of the intron with respect to the reading frame of the gene at the intron-exon boundaries [31]. Phase 0 introns fall between two codons whereas phase 1 and phase 2 introns fall at the termination of the first and the second base, respectively, of the final codon in the $5^{\prime}$ exon [31]. Intron phases are likely to be conserved since the modification of a frame at the proximal side of 


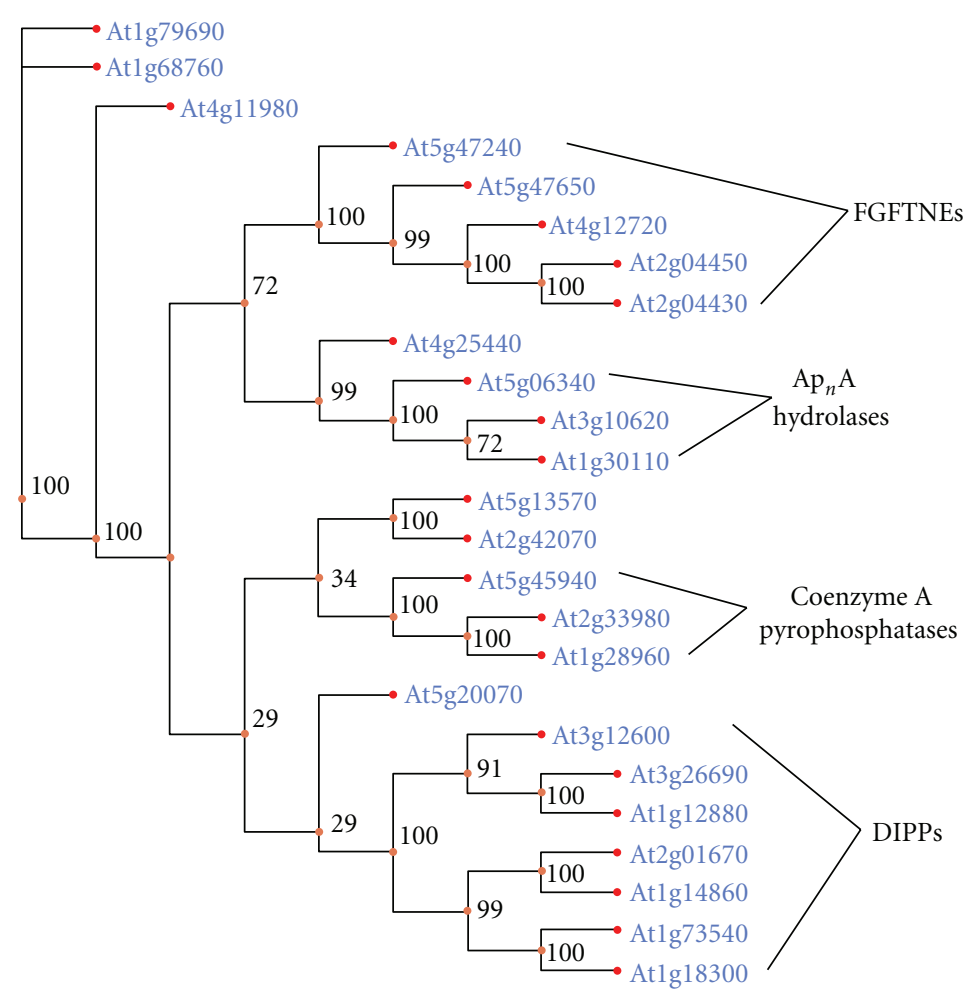

FIgURE 3: Maximum parsimony tree depicting the evolutionary relationships of the identified Nudix hydrolase genes in A. thaliana. Representative bootstrap values are shown as a percentage from 500 bootstrap replicates. The tree was rooted using the Atlg68760 gene.

an intron would necessitate a retaliatory change at the distal exon to maintain the reading frame $[31,32]$. In our study, the intron-exon structures of the Nudix hydrolases were obtained from the Xpro and the TAIR databases [20, 21]. A high degree of conservation of intron phases was detected in multimember families of the Nudix hydrolases (Table 3) as an indicator of extensive conservation of intron-exon architecture within these families. Such conservation of intron phases in families with multiple members is indicative of the above evolutionary difficulty in inducing frame-shifts within the genetic boundaries of a single gene. As further shown in Table 3, there are striking distinctions in the arrangement of introns and intron phases between families of the Nudix superfamily. It was concluded, based on the preservation of arrangement of intron phases within families and the striking dissimilarity of intron phase patterns between families, that there is a strong genetic basis for the subgrouping into families otherwise assigned by pairwise alignment.

3.4. Phylogenetic Analysis. Multiple sequence alignment of the 25 Nudix hydrolases was used as an entry for a phylogenetic analysis using DAMBE software. Protein level alignments were created using the CLUSTALW algorithm and converted to the corresponding DNA sequences for phylogenetic analysis. Gene trees were created based on the maximum parsimony method with bootstrap support from 500 replicates (Figure 3 ).

The outgroup used to root the gene trees was a compromise selected after assessing the occurrence of each of the 25 sequences between species seeking ancestral genes that were not prevalent in most eukaryotic lineages. Gene At1g68760 was used for tree rooting purposes, even though it cannot be considered a superlative outgroup, in terms of distinctness and ancestry. Even though homologues of this gene could be found in other eukaryotes, it was chosen as the outgroup due to its unique position as essential for plants but not for animals. It encodes a dihydroneopterin triphosphate hydrolase, an essential enzyme in the folate synthesis pathway in bacteria and plants [33]. Humans and other mammals lack a complete folate synthesis pathway and are dependent on folate from plant and bacterial sources [22]. In addition, the At1g68760 protein is the smallest member of the Nudix superfamily in Arabidopsis thaliana consisting of 147 amino acids. Due to the dependence of exon integration at the $5^{\prime}$ and $3^{\prime}$ end of proteins for evolutionary expansion in function, the At1g68760 protein serves as the smallest possible "core unit" to base the phylogenetic analysis.

The gene tree created using the maximum parsimony method shows the phylogenetic proximity of multimember families with the DIPPs, FGFTNEs, coenzyme A pyrophosphatases, and $\mathrm{Ap}_{n} \mathrm{~A}$ hydrolases forming monophyletic clusters (Figure 3). Further, there was high bootstrap support at nodes between members of a single family (Figure 3 ). Overall, the phylogenetic analysis was consistent with the classification of families obtained using pairwise alignments and intron phase analysis.

3.5. Motif Analysis. Conserved motifs in protein sequences that are indicative of functional sites and are conserved 
TABle 3: The distribution of exons and intron phases in multimember families of Nudix hydrolases in A. thaliana emphasizing the conservation in intron/exon architecture and of intron phase patterns within families.

\begin{tabular}{|c|c|c|c|}
\hline Family & Members & $\begin{array}{l}\text { Number of exons/size of protein } \\
\text { modules encoded by the exons } \\
(\mathrm{N} \rightarrow \mathrm{C})\end{array}$ & Intron phases $\mathrm{N} \rightarrow \mathrm{C}$ \\
\hline \multirow{3}{*}{$\mathrm{Ap}_{n} \mathrm{~A}$ hydrolases } & Atlg30110 & $5-22,17,31,31,74$ & $0,0,0,2$ \\
\hline & At3g10620 & $6-77,17,31,31,39,21$ & $0,0,0,2,0$ \\
\hline & At5g06340 & $6-76,17,31,31,39,33$ & $0,0,0,2,0$ \\
\hline \multirow{7}{*}{ DIPPs } & Atlg18300 & $2-63,144$ & 2 \\
\hline & Atlg73540 & $2-43,134$ & 2 \\
\hline & Atlg14860 & $4-23,36,28,89$ & $2,0,0$ \\
\hline & At3g12600 & $4-23,39,28,90$ & $2,0,0$ \\
\hline & Atlg12880 & $5-23,42,28,50,53$ & $2,0,0,0$ \\
\hline & At2g01670 & $5-28,35,28,43,48$ & $2,0,0,0$ \\
\hline & At3g26690 & $5-23,40,28,52,59$ & $2,0,0,0$ \\
\hline \multirow{5}{*}{ FGFTNEs } & At2g04430 & $8-50,22,46,26,17,11,38,73$ & $0,0,0,0,0,2,0$ \\
\hline & At4g12720 & $8-48,22,47,26,17,11,38,73$ & $0,0,0,0,0,2,0$ \\
\hline & At5g47650 & $8-47,22,46,27,17,11,37,62$ & $0,0,0,0,0,2,0$ \\
\hline & At5g47240 & $6-60,22,47,43,48,75$ & $0,0,0,0,0$ \\
\hline & At2g04450 & $8-48,22,47,26,17,11,37,75$ & $0,0,0,0,0,2,0$ \\
\hline \multirow{3}{*}{ Coenzyme A pyrophosphatases } & At5g45940 & $4-66,48,40,68$ & $1,0,0$ \\
\hline & Atlg28960 & $4-111,48,40,74$ & $1,0,0$ \\
\hline & At2g33980 & $6-105,48,40,66,30,13$ & $1,0,0,0,1$ \\
\hline
\end{tabular}

between functionally similar proteins [26] were sought within the Nudix superfamily. The conserved motifs detected in the Nudix hydrolases by the program MEME [23] are shown in Figure 4(a). Motif 1 in Figure 4(a) is the Nudix box, and by definition all 25 sequences of the Nudix superfamily in A. thaliana contained this motif.

As demonstrated in Figure 4(a), four other conserved motifs were found outside of the Nudix box in various members of the superfamily of Nudix hydrolases. Three of the identified motifs were unique to the DIPP (motif 2), FGFTNE (motif 3), and coenzyme A pyrophosphatase (motif 5) families, respectively. Such motifs are putative candidates for structural domains involved in positioning of the primary substrate of these families. Surprisingly, no motif unique to the fourth multimember family, the $\mathrm{Ap}_{n} \mathrm{~A}$ hydrolases, was detected in our analysis. Rather another motif (motif 4), which is present in all members of the FGFTNE family, was also found in the products of genes Atlg30110 and At5g20070, belonging to the $\mathrm{Ap}_{n} \mathrm{~A}$ hydrolase and $\mathrm{NADH}$ hydrolase families, respectively.

We have studied motif 4 in more detail. This motif is located in a region significant for substrate binding, at least in the Atlg30110 gene product, as shown by homology modeling of the Atlg30110 gene product with the structure of the lupin $\mathrm{Ap}_{4} \mathrm{~A}$ hydrolase (Figure 4(b)). The threedimensional structure of the $\mathrm{Ap}_{4} \mathrm{~A}$ hydrolase from Lupinus angustifolius was used in this comparison as the nearest such structure available from a plant enzyme [34]. Homology modeling positioned motif 4 in a region analogous to the highly mobile helix-loop-helix (helix 3-loop-helix 4), the preceding beta sheet, and a loop region that contained a metal-binding glutamate (Glu-125) in the lupin enzyme (Figure 4(b)). This highly mobile region was shown to be central to the binding of the nucleotide substrates in the lupin enzyme [35]. Helix 3, helix 4, and the linking loop region moved outward to accommodate the $\mathrm{ATP}-\mathrm{MgF}_{\mathrm{x}}$ complex at the substrate binding site [35]. The backbone and side chains of amino acids within the intervening loop region between helices 3 and 4 interacted with the adenine moiety of the ATP-MgF $\mathrm{F}_{\mathrm{x}}$ complex [35]. It is concluded that motif 4 is a potential substrate binding site that has evolved to accommodate different but perhaps related substrates in a range of Nudix families.

Though well separated in linear sequence from the Nudix box, motif 4 and the corresponding region in the lupin $\mathrm{Ap}_{4} \mathrm{~A}$ hydrolase are both placed in close proximity to the conserved catalytic helix within the Nudix motif in the 3D models (Figure 4(b)). Motif 4 in fact contains Glu-126 equivalent to the catalytically-essential glutamate Glu-125 in the lupin enzyme that contributes a ligand to an obligatory divalent cation at the active site [34]. This further emphasizes the potential of motif 4 to affect substrate hydrolysis.

3.6. Divergent Enzymes Containing Motif 4. Two enzymes annotated as hydrolases of $\mathrm{Ap}_{4} \mathrm{~A}$ (At1g30110) and NADH (At5g20070) and all members of the FGFTNE family contain motif 4. We performed bioinformatics analyses on the protein sequences containing motif 4 to investigate the putative functions of this promiscuous motif.

Bioinformatics analyses of the protein sequences of the At1g30110 and At5g20070 genes demonstrated distinctive features in both proteins. The Atlg30110 gene product in 


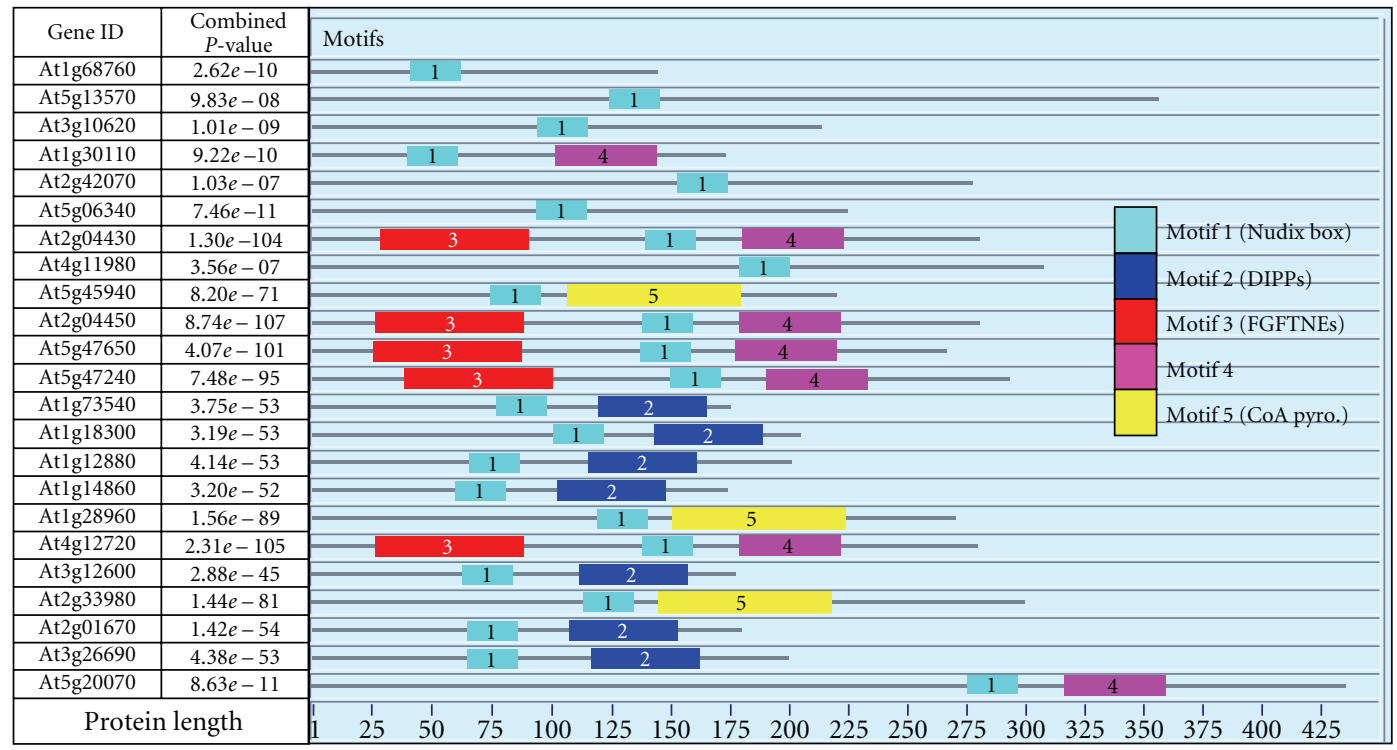

(a)

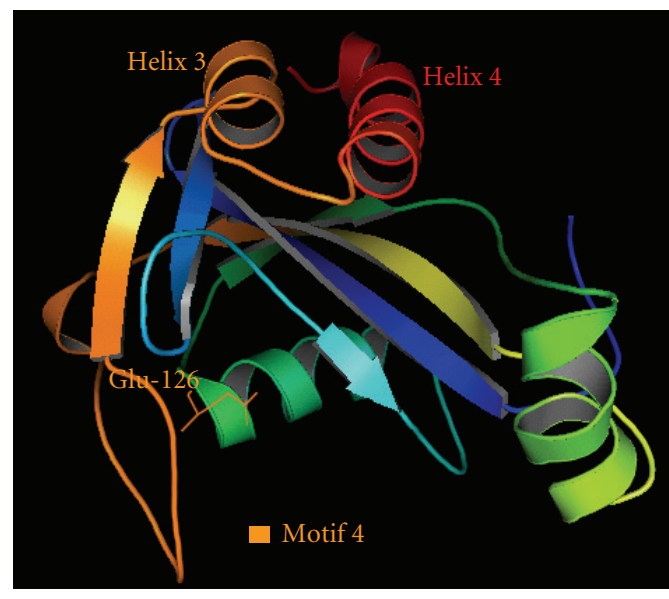

Atlg30110 homology model

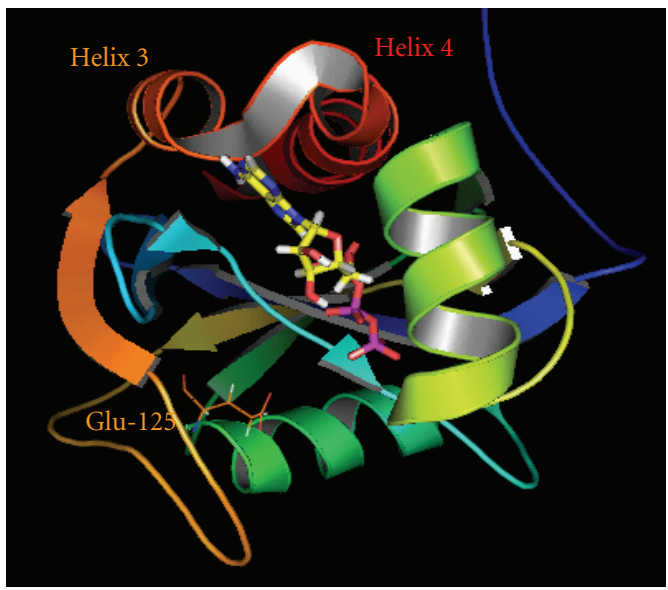

Lupin $\mathrm{AP}_{4}$ Ahydrolase

(b)

Figure 4: Conserved motifs in Arabidopsis Nudix hydrolases. (a) Different colored boxes and their designated numbers indicate separate and distinct motifs of 23 of the 25 Nudix hydrolases identified using MEME [23]. The At4g25440 and At1g79690 gene products were omitted from the figure for convenience of presentation. (b) Comparison of the homology model of the At1g30110 protein with that of the structure of the lupin $\mathrm{Ap}_{4} \mathrm{~A}$ hydrolase. The structure of the At1g30110 gene product (Left), a putative Ap $\mathrm{A}_{n} \mathrm{~A}$ hydrolase from A. thaliana modeled against the known structure of the lupin $\mathrm{Ap}_{4} \mathrm{~A}$ hydrolase (PDB ID-1JKN) in a complex with ATP-MgF $\mathrm{x}_{\mathrm{x}}$ (Right). Motif 4 is colored orange in the homology model, and helices 3 and 4 are marked. The loop region which contains the metal-binding Glu-125 in the lupin enzyme structure, the equivalent loop in the At1g30110 homology model, and the side chain of analogous Glu-126 are also depicted. The catalytic helix containing part of the conserved Nudix box is shown in green in each model.

particular appears to be quite distinct from all other plant $\mathrm{Ap}_{n} \mathrm{~A}$ hydrolases. Sequence alignment revealed differences that distinguished At1g30110 from other plant Nudix $\mathrm{Ap}_{4} \mathrm{~A}$ hydrolases, those from A. thaliana, Lupinus angustifolius, Hordeum vulgare, and Oryza sativa, none of which have a recognizable motif 4 (Supplementary-Figure A). Further, the Atlg30110 gene product lacks a large N-terminal extension, a likely preprotein region, found in all other plant $\mathrm{Ap}_{n} \mathrm{~A}$ hydrolases (Supplementary-Figure A). The inability to identify a marker motif common to all members of the $\mathrm{Ap}_{n} \mathrm{~A}$ hydrolases in $A$. thaliana from MEME analysis, is further evidenced that there is significant divergence of the At1g30110 gene product away from its Arabidopsis counterparts. Phylogenetic analysis of ten plant, bacterial, and animal Nudix $\mathrm{Ap}_{n} \mathrm{~A}$ hydrolases demonstrated that the Atlg30110 gene product holds a unique evolutionary niche: outside of the cluster of other plant Nudix hydrolases and close to the bacterial $\mathrm{Ap}_{n} \mathrm{~A}$ hydrolases (Figure 5). Analysis of gene duplication events also suggested that Atlg30110 originated prior to the division of the dicotyledons (A. thaliana) 


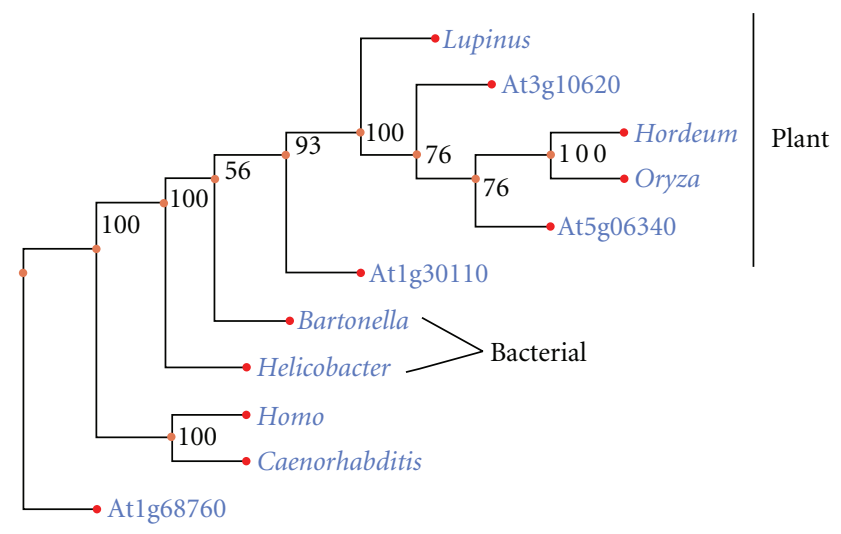

Figure 5: Phylogenetic analysis of Nudix $\mathrm{Ap}_{n} \mathrm{~A}$ Hydrolases. Maximum parsimony tree depicting the evolutionary relationships of plant (A. thaliana, L. angustifolius, O. sativa, H. vulgare), animal (Homo sapiens, Caenorhabditis elegans), and bacterial (Helicobacter pylori, Bartonella bacilliformis) $\mathrm{Ap}_{n} \mathrm{~A}$ hydrolase genes. Representative bootstrap values are shown as a percentage from 500 bootstrap replicates. The tree was rooted using the Atlg68760 gene encoding a dihydroneopterin triphosphate hydrolase from A. thaliana

TABLE 4: The summary of specific duplicated pairs of Nudix genes identified by assessing the segmental duplication events unearthed in the study by Blanc et al. [41]. The size of the proteins, the percentage of sequence identity between the pair of proteins, the segment length (number of genes), the designated family of Nudix genes, and the rough estimation of the age of the duplication event are also presented. "CoAPs" designates coenzyme A pyrophosphatases, and "Ap ${ }_{n} \mathrm{AHs}$ " is representative of $\mathrm{Ap}_{n} \mathrm{~A}$ hydrolases. In the study by Blanc et al. [41] the estimation of the age of the duplication event was conducted by the estimation of the synonymous substitution rates (Ks) between duplicated genes in sister regions. The duplications that are termed 'recent' contain median Ks values from 0.72 to 0.99 whereas those that are termed "old" have median Ks values from 1.82 to 6.03 .

\begin{tabular}{|c|c|c|c|c|c|c|c|}
\hline Gene 1 & Length of protein & Gene 2 & Length of protein & Sequence identity & Segment length & Family & Age \\
\hline Atlg18300 & 207 & Atlg73540 & 177 & $73 \%$ & 285 & DIPPs & Recent \\
\hline At1g14860 & 176 & At2g01670 & 182 & $78 \%$ & 53 & DIPPs & Recent \\
\hline At1g12880 & 196 & At3g26690 & 202 & $76 \%$ & 26 & DIPPs & Recent \\
\hline At1g28960 & 273 & At5g45940 & 222 & $50 \%$ & 8 & CoAPs & Old \\
\hline At3g10620 & 216 & At5g06340 & 227 & $51 \%$ & 2 & $\mathrm{Ap}_{n} \mathrm{AHs}$ & Old \\
\hline At4g12720 & 282 & At2g04430 & 283 & $54 \%$ & 3 & GFTNEs & Old \\
\hline
\end{tabular}

and monocotyledons (Oryza sativa) lineages (Figure 6). Gene Atlg30110 is likely to be an ancestral state of plant Nudix hydrolases from which the remaining $\mathrm{Ap}_{n} \mathrm{~A}$ hydrolase genes have undergone some degree of divergent evolution. Recent biochemical studies have demonstrated that the Atlg30110 gene product hydrolyzes $\mathrm{Ap}_{4} \mathrm{~A}$ as the preferred substrate in the presence of $\mathrm{Mn}^{2+}$ ions [36].

The 438 amino acid At5g20070 gene product contains the signature sequence SQPWPFP_S, found in all members of the NADH hydrolase family [14], and has been demonstrated to be active on both NADH and NADPH [38]. However, it has been shown that unlike other Nudix NADH hydrolases, which are localized to peroxisomes, the Arabidopsis counterpart is targeted to chloroplasts [38].

All members of the FGFTNE family, although resembling type 2 fibroblast growth factors, are involved in the hydrolysis of both ADP-ribose and NADH [39]. Fibroblast growth factors are regulatory peptides that are often secreted and belong to a separate protein superfamily [40]. The functional basis for the sequence similarity between the FGFTNEs and fibroblast growth factors is yet unknown. It is likely that signaling functions are associated with this Nudix family, and evidence from in vivo studies have already demonstrated that pleiotropic phenotypes result from the inactivation of a single FGFTNE gene $[11,12]$. In spite of several potential roles for FGFTNEs, their enzymatic capabilities to hydrolyze both NADH and ADP-ribose are of significance for the regulation of these molecules. The presence of motif 4 in all members of the FGFTNE family points to a role for this motif, in the hydrolysis of substrates containing an ADP moiety. Therefore, it appears that all proteins containing motif 4 are active on substrates such as $\mathrm{NADH}, \mathrm{ADP}$-ribose, and $\mathrm{Ap}_{4} \mathrm{~A}$ that contain a terminal $\mathrm{ADP}$ moiety.

\section{Discussion}

In this study, 25 Nudix hydrolases encoded by the genome of Arabidopsis thaliana were identified by the combination of hidden Markov model searches and manual refinement of search results. A similar attempt by Ogawa et al. [39] to identify the total complement of Nudix hydrolases in $A$. thaliana using the NCBI database did not include four of the sequences identified in this study. The same four enzymes were absent in the study by Bartsch et al. [12]. Neither the three members of the $\mathrm{Ap}_{n} \mathrm{~A}$ hydrolase family (Atlg30110, At3g10620, At5g06340) nor the mRNA decapping enzyme 

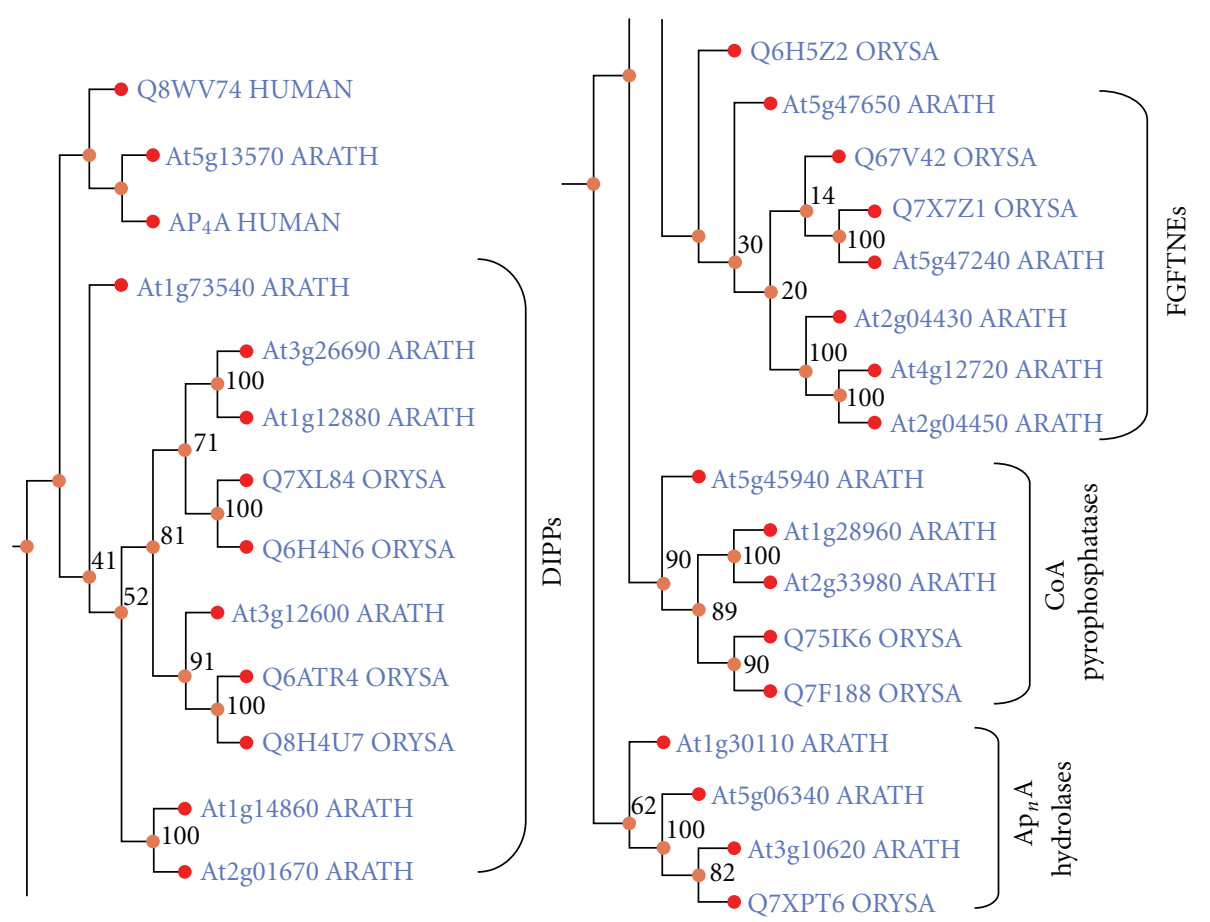

(a)

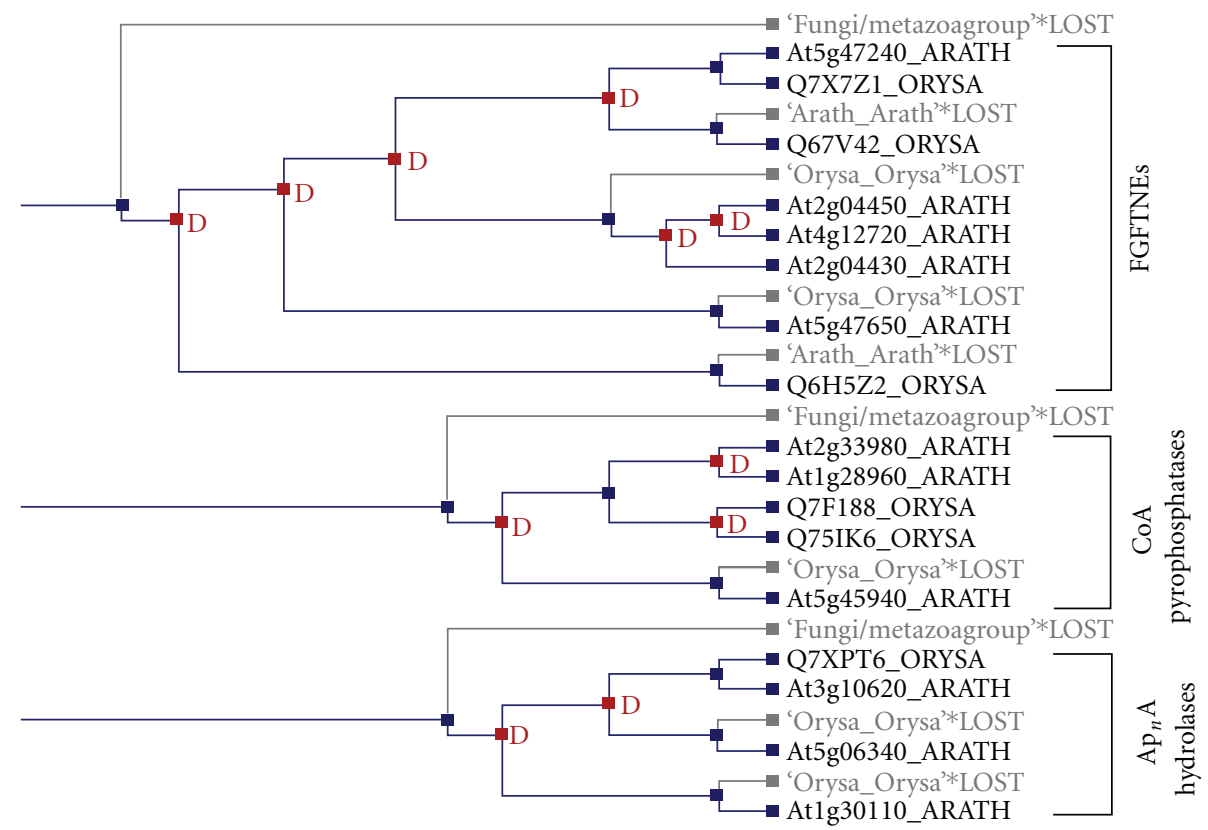

(b)

Figure 6: Gene duplication events in selective Nudix families. Maximum parsimony tree (selective regions shown adjacent to each other) depicting the phylogenetic relationships of Nudix hydrolase genes in the species A. thaliana, O. sativa, H. sapiens, S. cerevisiae, and S. elongatus rooted using the $\mathrm{NudC}$ gene from the gram negative bacterium Vibrio cholerae. Representative bootstrap values are shown as a percentage from 250 bootstrap replicates. ARATH and ORYSA are standard terminology for A. thaliana and O. sativa, respectively, according to the UniProt knowledgebase [37]. (b) Gene duplication events of the $A_{n}$ A hydrolase, coenzyme A pyrophosphatase, and FGFTNE families of Nudix hydrolases in A. thaliana. The gene tree from the species A. thaliana, O. sativa, H. sapiens, S. cerevisiae, and S. elongatus (Figure 6(a)) was reconciled with the corresponding binary species tree from NCBI as described in materials and methods section. $\boldsymbol{D}$ indicates a duplication event. 
(At5g13570) was identified in the studies by Ogawa et al. [39] and Bartsch et al. [12]. Further, three of the enzymes identified as Nudix hydrolases in Ogawa et al. [39] did not contain the conserved REXXEE sequence within the Nudix box. By contrast, the 25 Nudix hydrolases identified in our study contained the hexaresidue motif within the Nudix box sequence.

In a separate study by Muñoz et al. [10], 31 Nudix hydrolases were identified by database searches. However, six of the identified proteins did not fit the criteria for classical Nudix hydrolases, and unless biochemical evidence is presented to back up the claims, they cannot be annotated as Nudix enzymes. The debated Nudix enzymes are (1) gene product of At $2 \mathrm{~g} 04440$, which we have demonstrated earlier to be a product of a pseudogene; (2) At3g02780 and At5g16440 gene products, which are isopentenyl diphosphate isomerases lacking Nudix motifs; (3) gene products of At5g19460 and At19470, both of which lack the essentially conserved REXXEE hexaresidue sequence within the Nudix box. Ogawa et al. [38] demonstrated that gene products of At5g19460 and At19470 are incapable of hydrolyzing any of the classical Nudix substrates, with the exception of the diphosphoinositol polyphosphates, which were not tested in this study, further validating the claim that they fall outside of the Nudix superfamily; (4) gene product of At3g46200, which again lacks the essentially conserved REXXEE sequence, has not been demonstrated to be active on any Nudix substrate [39].

In summary, our survey confirms that, like most eukaryotic genomes, the Arabidopsis genome codes for multiple Nudix enzymes. Unicellular yeast encodes six Nudix hydrolases and the number of functional Nudix hydrolases in humans is at least 24 [15]. Furthermore, this study clearly demonstrates the power of HMMs to identify accurately all representative members of a mechanistically diverse protein superfamily and to minimize misannotations that can arise from manual database searches.

The identified Nudix hydrolases were classified into families by pairwise sequence alignments and the classification substantiated first using conserved genetic features such as intron phases and subsequently by assessing the phylogenetic proximity of individual family members. Of the identified families, the DIPPs and FGFTNEs in particular have expanded in A. thaliana compared to the representative members of these families in humans where four DIPPs and one FGFTNE have been reported [15]. Members of multimember families retained similar intron phases and were of a monophyletic origin, clearly demonstrating that the classification of families based on a 30\% sequence identity level was accurate in assigning members to particular families. Whether the same genetic architecture can be extended to include other plant and animal Nudix genes remain to be seen. Interspecies preservation of intronic phases within gene families is less likely since increases in gene number can be traced back to either whole genome duplication events or segmental gene duplications arising from a single genome. For example, from searching the data of the study by Blanc et al. [41], the increased presence of DIPPs in Arabidopsis thaliana was determined to have originated from recent segmental duplication events (Table 4).
According to the prevailing theory of enzyme evolution suggested by Petsko et al. [42], a majority of "new" substrate specificities are added on to the existing chemistry of catalysis by sequence divergences within substrate binding sites. Accordingly, the families of enzymes containing the catalytic Nudix motif in A. thaliana appear to have evolved to accommodate broad but familial substrate specificities as well as overlapping interfamilial substrate preferences, using modifications to their substrate binding sites. Computational motif analysis identified 4 motifs in Nudix enzymes in addition to the Nudix box that was by definition conserved between all identified members. Three of these were restricted to and present in all members of particular families. We conclude that motifs 2, 3, and 5 are markers of the DIPP, FGFTNE, and coenzyme A pyrophosphatase families, respectively.

One motif, motif 4 , was found in enzymes with annotated activities to $\mathrm{NADH}$ and $\mathrm{Ap}_{4} \mathrm{~A}$ substrates and in FGFTNEs. The product of the At1g30110 gene hydrolyzed $\mathrm{Ap}_{4} \mathrm{~A}$, and the At5g20070 gene product hydrolyzed NADH, whereas all members of the FGFTNE family were capable of hydrolyzing both ADP-ribose and NADH [36, 38, 39]. An ADP moiety is common to each of these substrates. Motif 4 in the At1g30110 gene product was mapped onto the substrate binding region suggesting that motif 4 directly or indirectly aids the binding of ADP-containing substrates to this region of the protein. Motif 4 appears to be a unique event of convergent evolution within the Nudix superfamily, where substrate binding sites of divergent proteins have evolved analogous motifs to accommodate overlapping or related substrate specificities.

\section{Conclusions}

We have demonstrated that HMM model-based genome scanning and MEME motif analysis have significantly improved the accuracy of identification and annotation of Nudix hydrolases encoded by the Arabidopsis genome. We conclude that deciphering the diversity, organization, and phylogeny of Nudix genes should facilitate future annotation of Nudix genes within sequenced genomes in other organisms. The utilization of intron-phases appears particularly relevant in assessing the evolution of gene families within this enzyme superfamily. Identification of motifs specific for three families and a fourth motif associated with particular substrate preferences suggests that similar analyses should aid in the assignment of function of new members of this mechanistically diverse protein superfamily in other organisms. Identification of the location of motif 4 adjacent to the catalytic site within the 3D structure of the Nudix enzyme has focused attention on this region of the protein as one undergoing evolution to allow access to differing substrates. In doing so, it highlights the potential importance of not only motif 4 but also the other family specific motifs 2, 3, and 5 as regions of the enzymes upon which to focus future structure-based studies and protein engineering efforts to understand the evolution of enzymatic activities. 


\section{Acknowledgment}

The authors are indebted to the Sir John and Lady Higgins Foundation for sponsoring the Ph.D. studies of DG.

\section{References}

[1] S. Sheikh, S. F. O’Handley, C. A. Dunn, and M. J. Bessman, "Identification and characterization of the Nudix hydrolase from the Archaeon, Methanococcus jannaschii, as a highly specific ADP-ribose pyrophosphatase," The Journal of Biological Chemistry, vol. 273, no. 33, pp. 20924-20928, 1998.

[2] W. Xu, P. Gauss, J. Shen, C. A. Dunn, and M. J. Bessman, "The gene $e .1$ (nudE.1) of T4 bacteriophage designates a new member of the Nudix hydrolase superfamily active on flavin adenine dinucleotide, adenosine $5^{\prime}$-triphospho- $5^{\prime}$-adenosine, and ADP-ribose," The Journal of Biological Chemistry, vol. 277, no. 26, pp. 23181-23185, 2002.

[3] W. Xu, C. A. Dunn, C. R. Jones, G. D’Souza, and M. J. Bessman, "The 26 Nudix hydrolases of Bacillus cereus, a close relative of Bacillus anthracis," The Journal of Biological Chemistry, vol. 279, no. 23, pp. 24861-24865, 2004.

[4] M. J. Bessman, D. N. Frick, and S. F. O'Handley, "The MutT proteins or "Nudix" hydrolases, a family of versatile, widely distributed, "housecleaning" enzymes," The Journal of Biological Chemistry, vol. 271, no. 41, pp. 25059-25062, 1996.

[5] M. E. Glasner, J. A. Gerlt, and P. C. Babbitt, "Evolution of enzyme superfamilies," Current Opinion in Chemical Biology, vol. 10, no. 5, pp. 492-497, 2006.

[6] C. Abeygunawardana, D. J. Weber, A. G. Gittis, et al., "Solution structure of the MutT enzyme, a nucleoside triphosphate pyrophosphohydrolase," Biochemistry, vol. 34, no. 46, pp. 14997-15005, 1995.

[7] J. Lin, C. Abeygunawardana, D. N. Frick, M. J. Bessman, and A. S. Mildvan, "The role of Glu 57 in the mechanism of the Escherichia coli MutT enzyme by mutagenesis and heteronuclear NMR," Biochemistry, vol. 35, no. 21, pp. 67156726, 1996.

[8] T. K. Harris, G. Wu, M. A. Massiah, and A. S. Mildvan, "Mutational, kinetic, and NMR studies of the roles of conserved glutamate residues and of lysine-39 in the mechanism of the MutT pyrophosphohydrolase," Biochemistry, vol. 39, no. 7, pp. 1655-1674, 2000.

[9] A. S. Mildvan, Z. Xia, H. F. Azurmendi, et al., "Structures and mechanisms of Nudix hydrolases," Archives of Biochemistry and Biophysics, vol. 433, no. 1, pp. 129-143, 2005.

[10] F. J. Muñoz, E. Baroja-Fernández, M. T. Morán-Zorzano, N. Alonso-Casajús, and J. Pozueta-Romero, "Cloning, expression and characterization of a Nudix hydrolase that catalyzes the hydrolytic breakdown of ADP-glucose linked to starch biosynthesis in Arabidopsis thaliana," Plant and Cell Physiology, vol. 47, no. 7, pp. 926-934, 2006.

[11] N. Jambunathan and R. Mahalingam, "Analysis of Arabidopsis Growth Factor Gene 1 (GFG1) encoding a Nudix hydrolase during oxidative signaling," Planta, vol. 224, no. 1, pp. 1-11, 2006.

[12] M. Bartsch, E. Gobbato, P. Bednarek, et al., "Salicylic acidindependent ENHANCED DISEASE SUSCEPTIBILITY1 signaling in Arabidopsis immunity and cell death is regulated by the monooxygenase FMO1 and the Nudix hydrolase NUDT7," The Plant Cell, vol. 18, no. 4, pp. 1038-1051, 2006.

[13] J. Xu, J.-Y. Yang, Q.-W. Niu, and N.-H. Chua, "Arabidopsis DCP2, DCP1, and VARICOSE form a decapping complex required for postembryonic development," The Plant Cell, vol. 18, no. 12, pp. 3386-3398, 2006.

[14] C. A. Dunn, S. F. O’Handley, D. N. Frick, and M. J. Bessman, "Studies on the ADP-ribose pyrophosphatase subfamily of the Nudix hydrolases and tentative identification of $\operatorname{trg} B$, a gene associated with tellurite resistance," The Journal of Biological Chemistry, vol. 274, no. 45, pp. 32318-32324, 1999.

[15] A. G. McLennan, "The Nudix hydrolase superfamily," Cellular and Molecular Life Sciences, vol. 63, no. 2, pp. 123-143, 2006.

[16] The Arabidopsis Genome Initiative, "Analysis of the genome sequence of the flowering plant Arabidopsis thaliana," Nature, vol. 408, no. 6814, pp. 796-815, 2000.

[17] S. R. Eddy, "Hidden Markov models," Current Opinion in Structural Biology, vol. 6, no. 3, pp. 361-365, 1996.

[18] J. D. Thompson, D. G. Higgins, and T. J. Gibson, "CLUSTAL $\mathrm{W}$ : improving the sensitivity of progressive multiple sequence alignment through sequence weighting, position-specific gap penalties and weight matrix choice," Nucleic Acids Research, vol. 22, no. 22, pp. 4673-4680, 1994.

[19] X. Xia and Z. Xie, "DAMBE: software package for data analysis in molecular biology and evolution," The Journal of Heredity, vol. 92, no. 4, pp. 371-373, 2001.

[20] E. Huala, A. W. Dickerman, M. Garcia-Hernandez, et al., "The Arabidopsis Information Resource (TAIR): a comprehensive database and web-based information retrieval, analysis, and visualization system for a model plant," Nucleic Acids Research, vol. 29, no. 1, pp. 102-105, 2001.

[21] V. Gopalan, T. W. Tan, B. T. K. Lee, and S. Ranganathan, "Xpro: database of eukaryotic protein-encoding genes," Nucleic Acids Research, vol. 32, database issue, pp. D59-D63, 2004.

[22] G. Basset, E. P. Quinlivan, M. J. Ziemak, et al., "Folate synthesis in plants: the first step of the pterin branch is mediated by a unique bimodular GTP cyclohydrolase I," Proceedings of the National Academy of Sciences of the United States of America, vol. 99, no. 19, pp. 12489-12494, 2002.

[23] T. L. Bailey and C. Elkan, "The value of prior knowledge in discovering motifs with MEME," in Proceedings of the $3 \mathrm{rd}$ International Conference on Intelligent Systems for Molecular Biology, vol. 3, pp. 21-29, Cambridge, UK, July 1995.

[24] T. Schwede, J. Kopp, N. Guex, and M. C. Peitsch, "SWISSMODEL: an automated protein homology-modeling server," Nucleic Acids Research, vol. 31, no. 13, pp. 3381-3385, 2003.

[25] K. Chen, D. Durand, and M. Farach-Colton, "NOTUNG: a program for dating gene duplications and optimizing gene family trees," Journal of Computational Biology, vol. 7, no. 34, pp. 429-447, 2000.

[26] E. L. L. Sonnhammer, S. R. Eddy, and R. Durbin, "Pfam: a comprehensive database of protein domain families based on seed alignments," Proteins: Structure, Function and Genetics, vol. 28, no. 3, pp. 405-420, 1997.

[27] C. J. Sigrist, L. Cerutti, N. Hulo, et al., "PROSITE: a documented database using patterns and profiles as motif descriptors," Briefings in Bioinformatics, vol. 3, no. 3, pp. 265274, 2002.

[28] N. Hulo, A. Bairoch, V. Bulliard, et al., "The PROSITE database," Nucleic Acids Research, vol. 34, database issue, pp. D227-D230, 2006.

[29] A. G. Murzin, S. E. Brenner, T. Hubbard, and C. Chothia, "SCOP: a structural classification of proteins database for the investigation of sequences and structures," Journal of Molecular Biology, vol. 247, no. 4, pp. 536-540, 1995.

[30] D. Gunawardana, H.-C. Cheng, and K. R. Gayler, "Identification of functional domains in Arabidopsis thaliana mRNA 
decapping enzyme (AtDcp2)," Nucleic Acids Research, vol. 36, no. 1, pp. 203-216, 2008.

[31] B. C. Meyers, A. Kozik, A. Griego, H. Kuang, and R. W. Michelmore, "Genome-wide analysis of NBS-LRR-encoding genes in Arabidopsis," The Plant Cell, vol. 15, no. 4, pp. 809834, 2003.

[32] N. Tijet, C. Helvig, and R. Feyereisen, "The cytochrome P450 gene superfamily in Drosophila melanogaster: annotation, intron-exon organization and phylogeny," Gene, vol. 262, no. 1-2, pp. 189-198, 2001.

[33] S. M. J. Klaus, A. Wegkamp, W. Sybesma, J. Hugenholtz, J. F. Gregory III, and A. D. Hanson, "A Nudix enzyme removes pyrophosphate from dihydroneopterin triphosphate in the folate synthesis pathway of bacteria and plants," The Journal of Biological Chemistry, vol. 280, no. 7, pp. 5274-5280, 2005.

[34] J. D. Swarbrick, T. Bashtannyk, D. Maksel, et al., "The threedimensional structure of the Nudix enzyme diadenosine tetraphosphate hydrolase from Lupinus angustifolius L," Journal of Molecular Biology, vol. 302, no. 5, pp. 1165-1177, 2000.

[35] J. I. Fletcher, J. D. Swarbrick, D. Maksel, K. R. Gayler, and P. R. Gooley, "The structure of $\mathrm{Ap}_{4} \mathrm{~A}$ hydrolase complexed with ATP- $\mathrm{MgF}_{\mathrm{x}}$ reveals the basis of substrate binding," Structure, vol. 10, no. 2, pp. 205-213, 2003.

[36] B. Szurmak, A. Wysłouch-Cieszyńska, M. Wszelaka-Rylik, W. Bal, and M. Dobrzańska, "A diadenosine 5', 5" $-\mathrm{P}^{1} \mathrm{P}^{4}$ tetraphosphate $\left(\mathrm{Ap}_{4} \mathrm{~A}\right)$ hydrolase from Arabidopsis thaliana that is activated preferentially by $\mathrm{Mn}^{2+}$ ions," Acta Biochimica Polonica, vol. 55, no. 1, pp. 151-160, 2008.

[37] M. Schneider, A. Bairoch, C. H. Wu, and R. Apweiler, "Plant protein annotation in the UniProt knowledgebase," Plant Physiology, vol. 138, no. 1, pp. 59-66, 2005.

[38] T. Ogawa, K. Yoshimura, H. Miyake, et al., "Molecular characterization of organelle-type Nudix hydrolases in Arabidopsis thaliana," Plant Physiology, vol. 148, no. 3, pp. 1412-1424, 2008.

[39] T. Ogawa, Y. Ueda, K. Yoshimura, and S. Shigeoka, "Comprehensive analysis of cytosolic Nudix hydrolases in Arabidopsis thaliana," The Journal of Biological Chemistry, vol. 280, no. 26, pp. 25277-25283, 2005.

[40] C. Popovici, R. Roubin, F. Coulier, and D. Birnbaum, "An evolutionary history of the FGF superfamily," BioEssays, vol. 27, no. 8, pp. 849-857, 2005.

[41] G. Blanc, K. Hokamp, and K. H. Wolfe, "A recent polyploidy superimposed on older large-scale duplications in the Arabidopsis genome," Genome Research, vol. 13, no. 2, pp. 137-144, 2003.

[42] G. A. Petsko, G. L. Kenyon, J. A. Gerlt, D. Ringe, and J. W. Kozarich, "On the origin of enzymatic species," Trends in Biochemical Sciences, vol. 18, no. 10, pp. 372-376, 1993. 

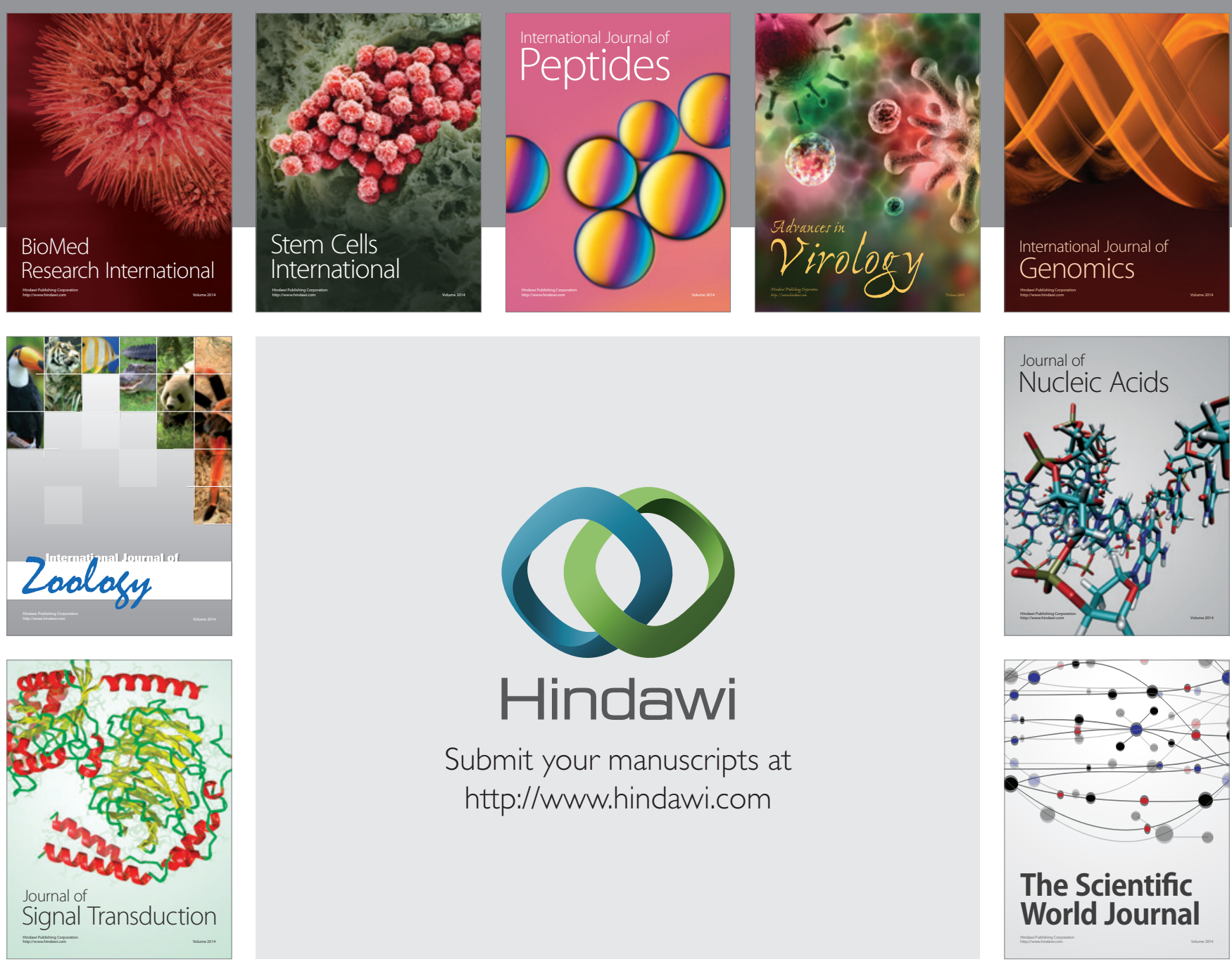

Submit your manuscripts at

http://www.hindawi.com
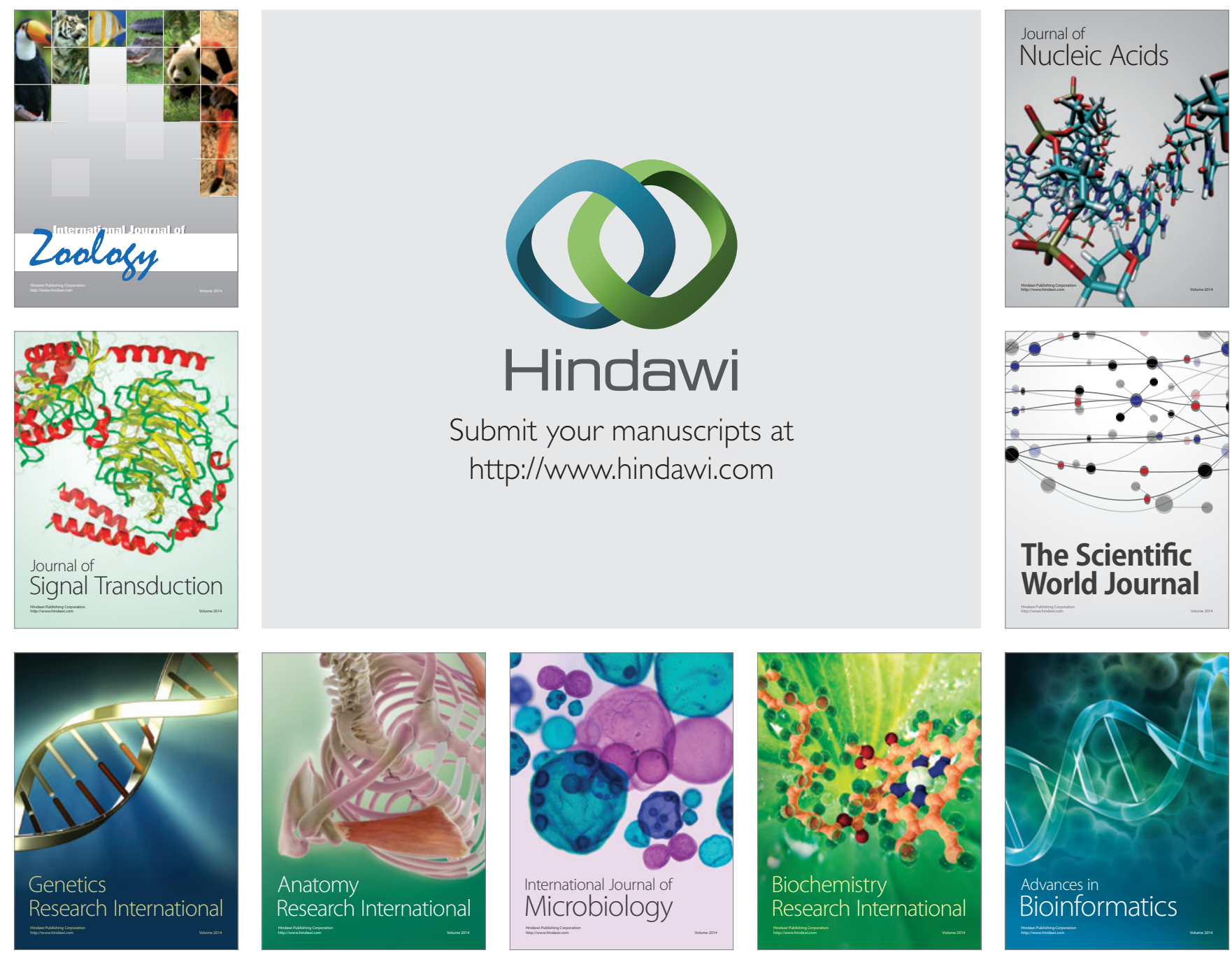

The Scientific World Journal
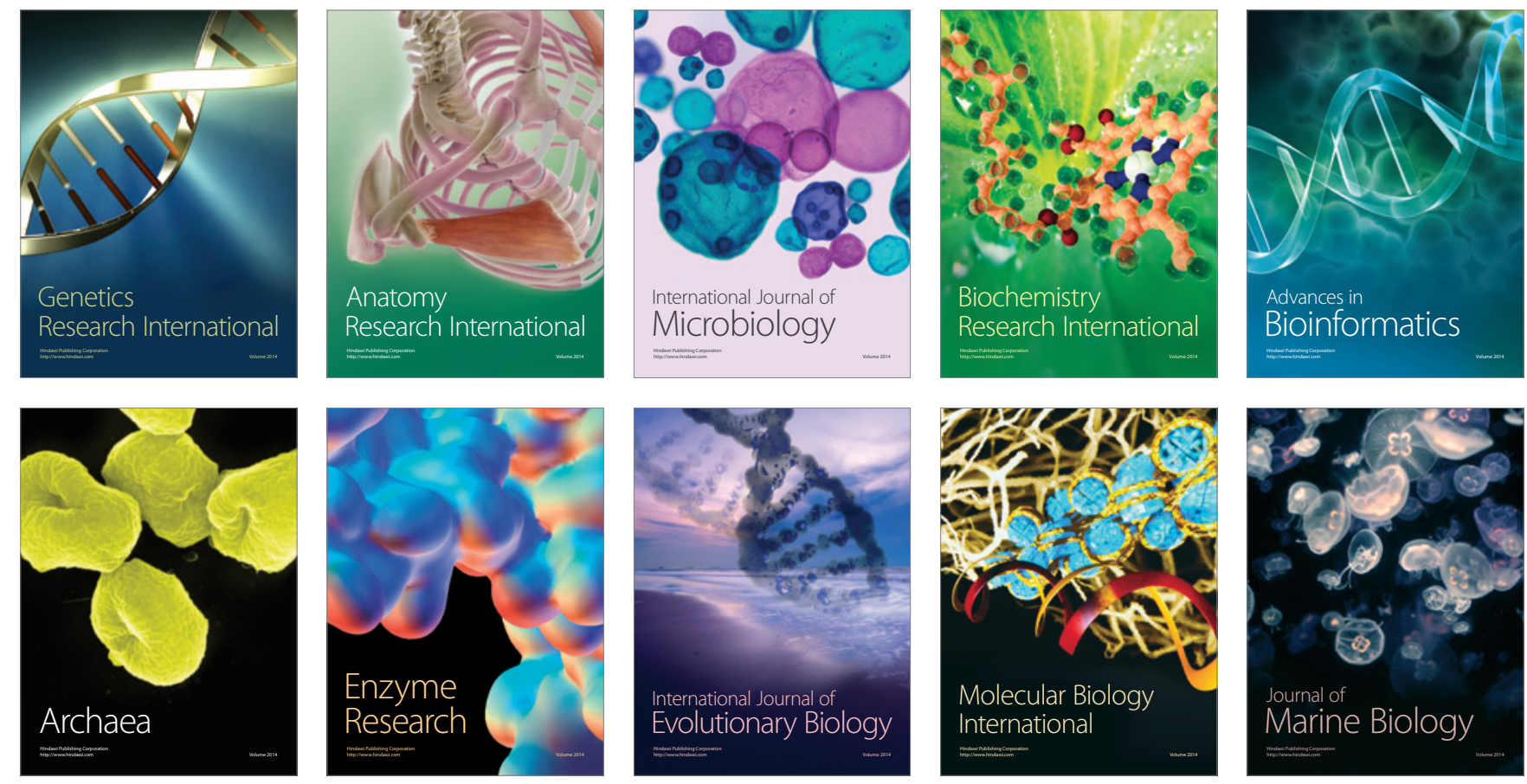